\title{
La hipótesis de la compresión de la morbilidad: un ejemplo de desarrollo teórico en epidemiología
}

Sergio López-Moreno, M.C., ${ }^{(1)}$ Alexánder Corcho-Berdugo, M.C., ${ }^{(1)}$

Malaquías López-Cervantes, M.C., Ph.D.11

\author{
López-Moreno S, Corcho-Berdugo A, \\ López-Cervantes $\mathrm{M}$. \\ La hipótesis de la compresión de la morbilidad: \\ un ejemplo de desarrollo teórico \\ en epidemiología. \\ Salud Publica Mex 1998;40:442-449.
}

\section{Resumen}

En este documento se revisa la hipótesis de la compresión de la morbilidad, propuesta por James Fries en 1980, co mo un ejemplo de elaboración teórica en el campo de la epidemiología. Se presentan inicialmente algunos rasgos fundamentales de las teorías científicas y se hace un recuento histórico de los modelos causales más importantes que ha utilizado la epidemio logía. Posteriormente, se presentan los fundamentos de la hipótesis de la compresión de la morbilidad, y se examinan sus posibilidades y limitaciones actuales, así como sus implicaciones epistemológicas con base en la teoría estructuralista. Se concluye que si el modelo de la compresión de la morbilidad fuera correcto, tendría una enorme capacidad para describir,explicar y predecir la salud y la enfermedad en las poblaciones humanas; por lo tanto, el desarrollo sistemático de este tipo de propuestas tiene una extraordinaria importancia para la salud pública.

Palabras clave: epidemiología; modelos teóricos; morbilidad; epistemología

\author{
López-Moreno S, Corcho-Berdugo A, \\ López-Cervantes $M$. \\ The hypothesis of the compression of morbidity: \\ An example of theoretical development \\ in epidemiology.
}

Salud Publica Mex 1998;40:442-449.

\section{A bstract}

In this paper we review a hypothesis put forth in 1980 by James Fries under the name of the compression of morbidity, as an example of theoretical development in the field of epidemiology. Initially we discuss some of the essential features of scientific theories and make a historical account of the most important causal models that epidemiology has embraced. Then, the basis for proposing that a compression of the morbidity is taking place are described focusing on its advantages and limitations when it is analyzed from the point of view of structuralism. Finally, we conclude that, to the extent that they are correct, models of this kind have the potential of describing and predicting the evolution of human health; hence, their systematic development entails an extraordinary importance for public health.

Key words: epidemiology; models, theoretical; morbidity; epistemology

\begin{abstract}
a enorme capacidad que las ciencias ganaron duL rante los tres últimos siglos para explicar el orden del mundo -orden que, por cierto, aspira a ser definitivo sin lograrlo- las ha colocado en un lugar semejante al que en el pasado tuvieron otros sistemas simbólicos como la mitología y la religión. Este lugar
\end{abstract}

de predominio se alcanzó desarrollando esquemas explicativos acerca de fenómenos sobre los cuales antes sólo teníamos argumentos mitológicos o religiosos. Aunque en algunos casos este desplazamiento ha sido un proceso lento, las ciencias han seguido ganando terreno y ha sido cuestión de tiempo para que el cono-

(1) Centro de Investigación en Sistemas de Salud, Instituto N acional de Salud Pública, México.

Fecha de recibido: 4 de marzo de 1998 - Fecha de aprobado: 24 de septiembre de 1998 Solicitud de sobretiros y correspondencia: Dr. Malaquías López Cervantes. Instituto N acional de Salud Pública, Av. Universidad N 0.655, colonia Santa María A huacatitlán, 62508 Cuernavaca, Morelos, México. 
cimiento científico se imponga sobre otros saberes, incorporándose al discurso cotidiano de los distintos grupos sociales.

El predominio de la explicación científica sobre el resto de explicaciones posibles es casi absoluto, de manera tal que hemos pasado de un mundo regido por la certeza religiosa, a otro caracterizado por la certeza científica. Hoy, tanto los procesos patológicos como las modificaciones del ambiente o de los mercados se explican, así sea provisionalmente, de acuerdo con los criterios de la ciencia; la educación se basa cada vez más en la descripción científica del mundo y un argumento vale tanto como la cantidad de ciencia que contenga. No es gratuito, entonces, que la cultura contemporánea gire en torno a las ciencias y que en las esferas política, económica y administrativa se les aprecie tanto.

Por lo anterior, resulta extraño que la filosofía de las ciencias sea un asunto que interese poco a los investigadores. Este desinterés resulta todavía más inquietante si tenemos en cuenta que, en la actualidad, las distintas disciplinas científicas buscan acceder a los recursos disponibles en forma proporcional a su grado de cientificidad. De ahí que la importancia de esta reflexión no sólo tenga consecuencias teóricas y de largo plazo, sino efectos prácticos casi inmediatos.

En el terreno de las ciencias de la salud, la cientificidad de la epidemiología se ha cuestionado reiteradamente. Esta situación ha motivado frecuentes quejas por parte de los epidemiólogos, pero pocos aportes sobre el carácter científico de la disciplina. En parte, esto se debe a que los avances teóricos más importantes de la epidemiología son relativamente recientes, pero también a la escasa importancia concedida por los mismos epidemiólogos a la reflexión teórica.

El presente documento tiene el propósito de participar en el debate acerca del papel de la teoría en la evolución de la epidemiología, proponiendo a la hipótesis de la compresion de la morbilidad como un ejemplo de esta elaboración teórica. Para facilitar la exposición se revisan primero algunos rasgos fundamentales de las teorías científicas y se hace un recuento histórico de los modelos causales más importantes que ha utilizado la epidemiología.

\section{Antecedentes}

\section{La naturaleza de las teorías científicas}

La concepción que durante largo tiempo dominó el panorama epistemológico sobre la construcción del conocimiento científico afirma que el desarrollo de las leyes y teorías científicas es resultado de la observación atenta de la realidad y la recolección meticulosa de datos, por parte de individuos dotados de la suficiente capacidad para ordenarlos, sistematizarlos y analizarlos. De este modo, la ciencia se presenta como un cuerpo sólido de conocimientos ciertos y estables, que se amplía y profundiza a medida que se realizan observaciones más precisas y con mejores instrumentos. Según esta concepción, que denominaremos genéricamente empirismo, la observación científica es la actividad a partir de la cual puede llegarse a las mejores certezas acerca del mundo. Para el empirismo, las ciencias nos conducen al conocimiento de la realidad tal cual es, ya que las teorías científicas son resultado de la observación y corresponden fielmente a los hechos sobre los que tratan. ${ }^{1}$ Sin embargo, aun aceptando la tajante distinción que el empirismo establece entre la observación y la teoría, un primer problema aparece ante la imposibilidad de que esta última explique de manera satisfactoria la totalidad de las observaciones que implica.

Sobre la separación teoría-observación, puede señalarse que la mayoría de las escuelas filosóficas aceptan formalmente tal distinción y que la postura adoptada al respecto ha servido, entre otros elementos, para diferenciarlas entre sí. Un segundo aspecto es la creencia de que las teorías reflejan fielmente las observaciones realizadas, ante lo cual es crucial mantener presente que los científicos generalmente utilizan la palabra observaciones para referirse al conjunto de las que han tomado en cuenta para llevar a cabo sus investigaciones; no obstante, cuando una teoría alcanza cierto grado de complejidad es imposible tener la certeza de que todas las observaciones pertinentes han sido tomadas en cuenta. Por lo tanto, jamás estaremos seguros de que la investigación ha considerado todas las observaciones que pueden resultar "útiles" a la teoría. Si sólo bastase la acumulación exhaustiva de observaciones para obtener buenas teorías, la investigación científica podría realizarse por medio de un sistema adecuado de cómputo, lo cual, como afirma Thuillier, ${ }^{1}$ resulta absurdo. Es evidente que la selección de las observaciones que un científico considera pertinentes nunca es arbitraria, ya que obedece a las necesidades que implica la teoría que ha elegido para desarrollar su trabajo.

Pueden señalarse tres consideraciones sobre la postura empirista. La primera es de carácter lógico y se refiere a que, al ser imposible que una teoría contenga todas las observaciones que implica, siempre existe la posibilidad de que algunas de ellas, no consideradas por el investigador, la contradigan; ésta es la razón por la que, aun entre las teorías científicas mejor establecidas, el conocimiento siempre se considera pro- 
visional. ${ }^{2}$ La segunda, de naturaleza metodológica, se refiere a la necesidad de desarrollar teorías que impliquen observaciones capaces de refutarlas, más que observaciones confirmatorias. ${ }^{3}$ La tercera alude a la necesidad de reconocer que las teorías científicas son estructuras de naturaleza incompleta y conflictiva, por lo que siempre existirán observaciones explicadas insuficientemente, o que contradigan a la teoría que debiera explicarlas. ${ }^{4}$ Los tres señalamientos se basan en la idea de que la adopción de una teoría precede a la selección de las observaciones que le son pertinentes y que el carácter científico de las observaciones está determinado por el grado de correspondencia que guardan con la teoría. ${ }^{1}$ Queda claro que para evaluar una observación antes debe evaluarse la teoría. Para ello, es necesario contar con una noción, aunque sea provisional, de la naturaleza de las teorías científicas, tanto para determinar su validez como representaciones de la realidad, cuanto para evaluar la pertinencia de su contrastación empírica.

El empirismo lógico, no obstante, pretendió durante la primera mitad de este siglo reconstruir todo el conocimiento científico, partiendo de la idea de que era posible elaborar un inventario de conocimientos básicos fundados en lo observable (es decir, en lo dado a los sentidos); con este criterio se intentó reducir el conocimiento teórico a sus bases observacionales, concibiendo las teorías como conjuntos de enunciados (teóricos y observacionales) lógicamente estructurados. Los enunciados teóricos serían aquellos que se refieren a eventos inobservables, y que se construyen obedeciendo reglas de deducción lógica. Los enunciados observacionales designarían propiedades observables de los objetos y los eventos, por lo cual serían los únicos del todo seguros. En esta línea de pensamiento, los enunciados teóricos tendrían validez sólo al conectarse con los enunciados observacionales.

Durante la segunda mitad del siglo XX, los filósofos racionalistas criticaron severamente el empirismo lógico, desarrollando novedosas concepciones acerca de las teorías científicas. ${ }^{5}$ Entre estas corrientes destacan la teoría estructural de Suppes y Sneed ${ }^{6,7}$ y el historicismo de Thomas Kuhn. ${ }^{8}$ Los primeros demostraron las ventajas que ofrece el concepto de modelo para el estudio teórico de las ciencias, mientras que el último terminó de exhibir las limitaciones del empirismo, que insistía en considerar el desarrollo científico como un proceso caracterizado por la acumulación de conocimientos sin discontinuidades o rupturas, demostrando la incapacidad de esta corriente para dar cuenta de las revoluciones científicas y de sus diferencias con lo que él mismo denominó "periodos de ciencia normal". La escuela epistemológica fundada por estos autores, influidos fuertemente por la naciente epistemología genética de Jean Piaget, puede llamarse genéricamente estructuralismo.

Para los estructuralistas, la mejor forma de desplegar una teoría es mediante la representación de su modelo general, el cual debe representar a todos los modelos potenciales posibles acerca de lo que la teoría dice, satisfaciendo sus leyes fundamentales. Además de enunciados conceptuales y observacionales, una teoría debe contener el conjunto de condiciones que permitan su ligadura, los diversos modelos potenciales de la teoría y el conjunto de condiciones en donde ésta pueda aplicarse. Para esta escuela, la importancia de un enunciado obedece al lugar que ocupa dentro de la estructura general de la teoría y a las relaciones que establece con el resto de sus componentes. Dado que estas relaciones pueden ser cambiantes, de acuerdo con el modelo potencial que cada escuela científica elija desarrollar, las relaciones presentes en la estructura de la teoría habitualmente resultan ser más importantes que los mismos enunciados.

Esta nueva concepción filosófica sobre las ciencias resume en cierta medida la historia de la ciencia occidental. En la primera etapa del desarrollo científico, imperaba una visión de la realidad basada en la búsqueda de la esencia de los objetos; los fenómenos se definían por sus aspectos sustanciales, y las relaciones que establecían con el resto del mundo eran consideradas un producto de sus cualidades. En una segunda etapa, la ciencia centró su actividad en la búsqueda de las relaciones existentes entre las cosas. Los objetos de estudio pasaron a ser vistos como partes de procesos determinados por las relaciones que establecían con el resto del mundo; para explicarlos debieron generarse modelos dinámicos, cuya variable fundamental era el tiempo. En esta fase se otorgó a la medición un papel fundamental en la actividad científica, y se llegó a pensar que la cuantificación era la mejor, o acaso la única manera, de evaluar las relaciones entre los procesos estudiados por las ciencias. La tercera etapa del desarrollo científico se inició en este siglo, al florecer la teoría de la homeostasis y los modelos explicativos de los procesos de autogobierno, autopreservación y autorreproducción de los sistemas naturales. ${ }^{9}$ En esta última fase, la principal actividad de las ciencias se ha centrado en el estudio de los sistemas complejos y de las relaciones presentes en ellos. La teoría estructural es el paradigma filosófico de este periodo.

\section{El desarrollo téorico de la epidemiología}

Esta disciplina ha experimentado durante su desarrollo un proceso similar al del resto de las ciencias natu- 
rales. Los cimientos de la epidemiología moderna datan del siglo XVII, cuando se desarrollaron las primeras técnicas descriptivas de medición de la enfermedad en el ámbito poblacional; ${ }^{10}$ desde el siglo XVIII y hasta la primera mitad del siglo XIX, la epidemiología se adhirió firmemente a la teoría miasmática, que fue el pensamiento dominante entre los salubristas europeos. En 1839 William Farr propuso los conceptos de poblacion en riesgo, grupo de comparación y duracion de la exposicion; tres lustros después, para demostrar que el cólera era transmitido por el agua, John Snow aplicó un método cuantitativo, con el que se estudiaron todos los brotes y epidemias infecciosas durante los siguientes cincuenta años.

Hacia finales del siglo XIX y principios del XX, siguiendo las pautas marcadas por el desarrollo científico biomédico, la epidemiología adoptó la teoría del germen. Esta teoría, reproduciendo el modelo de trenes causales de la física, afirmaba que para cada efecto existe una sola causa y que ambos eventos se relacionan siguiendo patrones constantes, en forma de cadenas lineales. Los seguidores de esta teoría fueron particularmente exitosos en la identificación de la etiología de muchas enfermedades e incluso en su control. El hecho de que las enormes consecuencias de las enfermedades transmisibles fueran, en gran parte, controladas en los hoy países desarrollados, dio una enorme credibilidad a este paradigma. No obstante, la teoría del germen condujo finalmente a un callejón sin salida, ya que la estrecha perspectiva de relacionar agentes simples, uno a uno con enfermedades específicas, fue incapaz de proponer y encontrar soluciones para aquellas enfermedades que no son necesariamente producidas por un germen. Además, la búsqueda frenética de los microrganismos retrasó el avance de la epidemiología, ya que resultaba insensato plantear investigaciones que no se basaran en un enfoque microbiológico.

A partir de la segunda guerra mundial, en la mayor parte de los países desarrollados, la mortalidad por enfermedades crónicas comenzó a superar a la producida por las enfermedades infecciosas agudas. La enfermedad coronaria y el cáncer de pulmón aumentaron lo suficiente para que se consideraran verdaderas epidemias. Al intentar entender y controlar este nuevo tipo de problemas de salud, los epidemiólogos se enfrentaron con circunstancias desconocidas, que requerían nuevos planteamientos respecto a su etiología. Al principio, se realizaron estudios descriptivos y exploratorios, tratando de encontrar pistas acerca de la causalidad a partir de la contrastación de los perfiles de estas enfermedades en distintos grupos humanos y en distintos lugares y momentos. Conforme se fueron identificando características o factores que, en forma recurrente, se relacionaban con la prevalencia, incidencia o mortalidad debidas a algún padecimiento, los epidemiólogos requirieron de propuestas conceptuales cada vez más complejas para buscar y proponer las causas de estas nuevas enfermedades y las epidemias por ellas producidas.

En 1950, R. Doll y B. Hill publicaron los resultados de un estudio de casos y controles, utilizado para determinar si existía relación entre el consumo de tabaco y la incidencia de cáncer pulmonar. Con este trabajo se aceptó y luego se popularizó la posibilidad de evaluar en forma retrospectiva el efecto de ciertas exposiciones, al comparar grupos de enfermos y no enfermos sin tener que esperar por años a que se desarrollara la enfermedad entre los sujetos expuestos a un presunto factor causal. Por otra parte, la utilización del diseño de cohortes, que se basa en el seguimiento de individuos sanos pero expuestos al factor presuntamente etiológico, se generalizó como resultado del éxito que obtuvo el ahora conocido como estudio Framingham, que se inició en 1945 y ha permitido conocer las relaciones que una amplia variedad de factores guardan entre sí y con la enfermedad coronaria.

Durante el periodo que va desde la posguerra hasta la actualidad, la epidemiología aceptó la posibilidad de que existieran múltiples causas para cada proceso patológico, y postuló que el peso causal de cada factor dependía de su cercanía con el presunto efecto, en el marco de una compleja red de causalidad. Este concepto de multicausalidad en forma de red fue introducido por B. MacMahon y se ha convertido en un elemento central de la epidemiología moderna. ${ }^{11}$ Una de sus principales ventajas radica en haber abierto el camino para el desarrollo y la aplicación de métodos estadísticos con un nivel de complejidad impensable en épocas previas, como es el caso de las técnicas de análisis multivariado.

Una versión más acabada del concepto de multicausalidad es la que propone que las relaciones establecidas entre las condiciones participantes en el proceso son tan complejas, que forman una unidad imposible de conocer completamente. Lo anterior corresponde al denominado modelo de la caja negra, que es una metáfora útil para representar un fenómeno cuyos mecanismos internos están ocultos al observador, por lo cual sugiere que la epidemiología debe limitarse a la búsqueda de aquellos elementos de la red que eventualmente permitan romper la cadena causal, haciendo innecesaria la identificación de todos los componentes del proceso de producción de la enfermedad. Este modelo tiene como principal limitación la dificultad para distinguir entre los determinantes individuales y 
los poblacionales de la enfermedad (es decir, entre las causas de los casos individuales y las causas de la incidencia). G. Rose advierte esta falta de discriminación al preguntarse si, por ejemplo, la presencia de hipertensión arterial en algunos individuos obedece a las mismas causas por las que algunas poblaciones tienen mayores tasas de hipertensión que otras. ${ }^{12}$

Más recientemente, otras corrientes han propuesto una alternativa denominada modelo historico-social. Este modelo plantea que la adopción mecánica de la multicausalidad ha desvirtuado el carácter social de la enfermedad, ya que concede el mismo peso a factores que son de naturaleza y jerarquía distintas. Además, cuestiona que el componente biológico de los procesos de salud colectiva siempre tenga un carácter determinante, por lo cual propone reexaminar estos fenómenos a la luz de su determinación histórica, económica y política. ${ }^{13}$ Según esta interpretación, la única forma de negar la naturaleza social de la enfermedad es admitiendo su génesis y resolución en sujetos completamente aislados de su entorno.

Desde nuestro punto de vista, tanto el enfoque basado en la simple enumeración y cuantificación de factores de riesgo, como el énfasis exclusivo en la dimensión social, conducen a interpretaciones restringidas acerca de la enfermedad y sus causas, limitando en forma innecesaria el papel de la epidemiología. En la primera de estas posturas, la búsqueda frenética de factores de riesgo genera perspectivas de un solo plano, que impiden entender las jerarquías inherentes a los factores estudiados; así, el papel de la epidemiología se torna eminentemente pragmático y no trata de explicar el proceso en su totalidad, sino que se restringe a la identificación de aquellos factores sobre los que es posible influir para romper la cadena causal, enfocándose en características individuales supuestamente modificables, pero olvidándose del origen social y ambiental de tales factores. Por otra parte, al sobrestimar la dimensión social, la segunda postura también toma la parte por el todo, reduciendo el estudio de la enfermedad y sus determinantes a las esferas económica y política, con lo cual se niega o se minimiza el hecho de que solamente ciertos individuos, pero no todos los que integran un estrato social, habrán de desarrollar una cierta enfermedad.

Lo comentado hasta aquí hace pensar que existe una serie de trabajos bien desarrollados acerca de los aspectos teóricos que sustentan a las corrientes señaladas; no obstante, los avances teóricos en el campo de la epidemiología han sido escasos, pues se ha brindado mucho más interés y esfuerzo al desarrollo de los aspectos metodológicos y técnicos de la disciplina. ${ }^{14,15}$ Entre los pocos trabajos realizados destacan aquellos que discuten la aplicación de las propuestas de Karl Popper en la investigación epidemiológica; el punto de partida fue el planteamiento formulado por vez primera por Carol Buck en 1975, mismo que dio origen a un acalorado debate que hasta la fecha subsiste. ${ }^{16-20}$

Entre las pocas obras que versan sobre los modelos de causalidad y los fundamentos epistemológicos de la epidemiología, destaca la de M. Susser, ${ }^{21}$ quien ve la enfermedad como un fenómeno poblacional que tiene lugar en múltiples niveles sistémicos, interconectados y jerarquizados. Contrariamente a la interpretación universalista, este autor plantea que los fenómenos colectivos de salud tienen lugar de manera similar a un conjunto de cajas chinas, es decir, siguiendo un arreglo tal que un sistema contiene a otro subordinado, y los cambios en un nivel influyen sobre el subsistema correspondiente, pero no necesariamente sobre la totalidad del arreglo. De esta manera, las relaciones existentes en cada nivel, del molecular al social, son válidas únicamente para explicar estructuras de complejidad y jerarquía similares; es decir, no pueden ser generalizadas a otros niveles. Con esta propuesta, que denomina ecoepidemiología, Susser descarta al universalismo como fundamento de la investigación epidemiológica. El ecologismo de Susser se refiere a la necesidad de limitar las generalizaciones de los sistemas biológico, humano y social a los nichos que les son propios.

Paralelamente a los debates abiertos por Buck y Susser, se han hecho otros esfuerzos para proponer y discutir los fundamentos conceptuales de la epidemiología y sus métodos. En este contexto de revisión conceptual surgió la hipótesis de la compresion de la morbilidad, que se presenta a continuación.

\section{La hipótesis de la compresión de la morbilidad}

En un artículo publicado en 1980 por The New England Journal of Medicine, James Fries hizo notar el hecho de que, aunque la esperanza de vida al nacimiento (EVN) se ha incrementado constantemente en todas las sociedades, no se podría sostener la idea de que tal ganancia fuera perpetua e ilimitada. ${ }^{22}$

Si desde el punto de vista estrictamente biológico fuese posible incrementar ilimitadamente la EVN del hombre, entonces ya habríamos podido observar que aquellos individuos genéticamente más aptos sobreviven mucho más tiempo que el común de las personas. ${ }^{23-25}$ En cambio, los datos disponibles indican que la proporción de centenarios no ha variado a lo largo del último siglo, a pesar del enorme incremento obte- 
nido hasta ahora en la EVN. ${ }^{26}$ Por otro lado, al analizar las curvas de supervivencia de cohortes específicas, se observa que los incrementos en la esperanza de vida (EV) disminuyen conforme avanza la edad. En Francia, de 1807 a 1971, la EVN se incrementó en 35 años, mientras que para el grupo de 65 y más años de edad el aumento de la EV fue de sólo 3.8 años. ${ }^{27}$ De manera similar, en Estados Unidos de América (EUA), de 1900 a 1980, la EVN se incrementó a razón de 0.35 años por año transcurrido, pero el aumento de la EV para los mayores de 65 años fue menor a 0.05 años por año, $y$ entre los mayores de 75 años apenas se incrementó en 0.008 años por año. ${ }^{28} \mathrm{~A}$ partir de la séptima década de la vida el indicador prácticamente ya no cambia, es decir, la ganancia anual en EV resulta casi imperceptible y este proceso es similar en todos los países. ${ }^{29}$

Fries señala que si la EVN continuara creciendo al mismo ritmo que hasta ahora, en algún momento del siglo próximo excedería la edad promedio de muerte proyectada para los ancianos. Lo anterior implica que, en algún momento, las cohortes de recién nacidos tendrán que desacelerar el ritmo con el que aumentan su $\mathrm{EV}$, o que las cohortes de ancianos tendrán que incrementarlo. Como ya se anotó, este último proceso difícilmente tendrá lugar, ya que es casi imposible ganar más años de vida para los grupos de mayor edad. Así nos encontramos frente al problema de identificar el límite potencial de la vida humana; a este respecto, mediante la extrapolación de las EV observadas en el último siglo, Fries indica que es posible encontrar un punto en el tiempo correspondiente al cruce de las líneas proyectadas de ganancia en la EV para distintas edades; en ese punto ya no cabría esperar un incremento ulterior de la EV, por lo que debiera considerarse como el promedio máximo de vida potencial. ${ }^{22} \mathrm{Al}$ comparar las proyecciones de la EVN con las EV de los grupos de 20, 65 y 75 años, Fries encontró que la intersección oscila entre 82.6 y 85.6 años de edad; así pudo plantear que el promedio máximo de vida potencial para el ser humano es de alrededor de 85 años. ${ }^{30}$

Si se grafica la distribución de las edades de muerte de una cohorte específica desde el nacimiento, hasta la muerte del último de sus miembros, los momentos del nacimiento y el límite máximo de vida potencial (maximum life span) corresponden, respectivamente, a sus extremos derecho e izquierdo, y la EVN, al promedio de la distribución. La EVN, el promedio máximo de vida y el límite máximo de vida potencial para la cohorte nacida en 1900 en EUA fueron de 47, 85 y 111 años, respectivamente. Al comparar estas cifras con las proyectadas para la cohorte nacida en 1980, se obtiene una gráfica como la que se muestra en la figura 1.

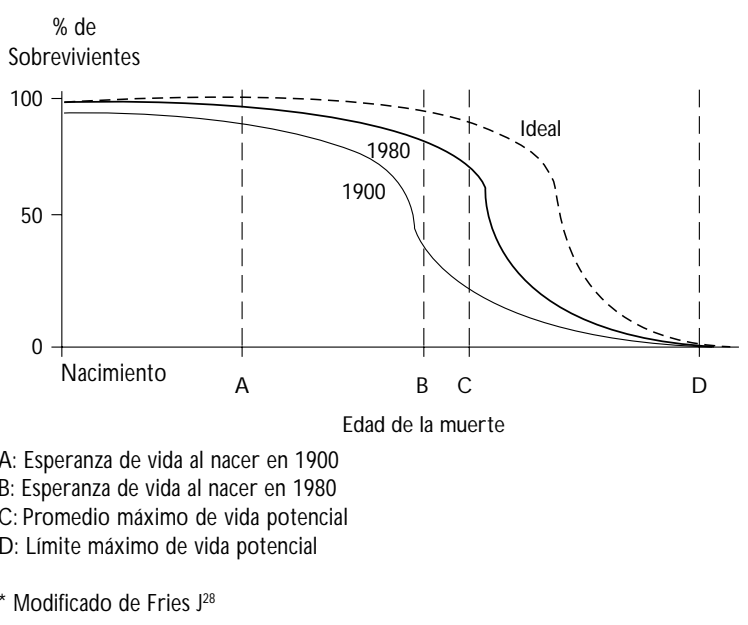

Figura 1. Curva de supervivencia para tres cohortes. Estados Unidos de América, 1900-1980*

En esta figura puede observarse que, a pesar de que la EVN se incrementó de 47 a 75 años entre 1900 (A) y 1980 (B), ni el promedio máximo de vida potencial (C) ni el límite máximo de vida potencial (D) variaron en forma significativa, ya que estos últimos indicadores mantuvieron valores de 85 y 111 años, respectivamente. Es conveniente hacer notar que las curvas seguirán declinando poco antes de los 85 años y que la proporción de centenarios no se incrementará. Una tercera curva indicaría el comportamiento ideal de la cohorte, suponiendo que fuese posible abatir al máximo las muertes prematuras y posponer la aparición de las enfermedades crónicas. Esta etapa, que corresponde al mayor acercamiento posible entre los puntos A y $\mathrm{C}$ de la gráfica, ha sido denominada la fase de rectangularización de las curvas de supervivencia.

Antes de revisar las implicaciones de la hipótesis de la compresión de la morbilidad, conviene señalar sus presupuestos. Estos son los siguientes: a) la ganancia en la EVN obtenida durante la primera mitad del presente siglo ha sido resultado de la desaparición de las muertes agudas, neonatales e infantiles; b) la morbilidad por enfermedades agudas ha sido sustituida por la morbilidad secundaria a enfermedades crónicas, y c) este proceso de sustitución de enfermedades ya ha concluido y los padecimientos crónicos han comenzado a declinar. ${ }^{22}$

Las principales implicaciones de la hipótesis son, en resumen, las siguientes: a) la rectangularización de las curvas de supervivencia obedece a la posposición de la edad de inicio de las enfermedades crónicas, combinada con la imposibilidad de aumentar el promedio 
máximo de vida potencial; b) en consecuencia, estas enfermedades tienden a concentrarse al final de la vida, y c) el tiempo de vida saludable (previo a la aparición de la enfermedad) tiende a ser mayor. Estos postulados constituyen la base de la hipótesis de la compresión de la morbilidad.

\section{Discusión}

Aunque el modelo de compresión de la morbilidad es de gran sencillez, a la fecha no ha sido sometido a contrastaciones empíricas suficientes. La mayor parte de la información empleada para proponerlo proviene de un país en donde ciertas enfermedades crónicas han disminuido, sin una explicación aceptada por la mayoría de los investigadores. Por lo tanto, es posible que en su formulación existieran sesgos no detectables bajo las condiciones en que fue establecido.

Desde el punto de vista empírico, un análisis detallado del modelo también permite ver que Fries partió de supuestos que no necesariamente se cumplen en todos los casos. Destaca la suposición de que la EV aumenta, fundamentalmente, como consecuencia de un desplazamiento de la edad promedio en que inician las enfermedades crónicas. En la mayoría de los casos esto no sucede así, ya que tal ganancia obedece, sobre todo, a las acciones (médicas y no médicas) que son emprendidas para evitar la muerte de los enfermos crónicos. De esta manera, no sólo es imposible sostener que el desplazamiento de la edad de inicio es el único mecanismo de incremento de la $\mathrm{EV}$, sino que posiblemente ni siquiera sea el más importante. La principal consecuencia de este hecho consiste en la posibilidad de que las fases de desplazamiento de la morbilidad hacia la derecha sean precedidas por una fase en donde la EV se incrementa por otras causas, dando como resultado una expansión de la morbilidad.

Un desplazamiento de la EV, que no corresponda con otro similar de la edad promedio de inicio de la enfermedad, provocaría en realidad una expansión de la zona de enfermedad. Este incremento en la EV puede ser consecuencia de un mejor tratamiento de las enfermedades crónicas, sin que se haya logrado evitar su aparición temprana. En la mayoría de los casos tal ha sido el mecanismo de incremento de la EV de los pacientes diabéticos, hipertensos, cardiovasculares y cancerosos. ${ }^{31}$ Aunque el mismo Fries y la mayor parte de sus críticos han analizado esta posibilidad, ${ }^{32,33} \mathrm{el} \mathrm{mo-}$ delo fundamental no ha sido modificado.

Por otro lado, de acuerdo con el modelo propuesto por Fries, la muerte natural tendría que irse convirtiendo en la causa más frecuente de muerte; sin embargo, empíricamente, este fenómeno no se ha observado. ${ }^{34,35}$ El modelo tampoco especifica qué sucederá mientras no sea posible culminar la etapa de sustitución de las enfermedades agudas (como sucede en los países pobres) y se enfrente un patrón mixto de mortalidad prematura y la debida a las enfermedades crónicas. ${ }^{36,37}$ En la mayor parte del mundo la evolución de la morbilidad ha sido distinta a la que predijo inicialmente la teoría de la transición epidemiológica, de tal manera que las enfermades agudas han permanecido (y en ocasiones aumentado), aunque el volumen de enfermedades crónicas y accidentes se ha incrementado. ${ }^{*}$ Finalmente, es probable que el proceso compresivo siga un comportamiento diferente en cada enfermedad (que también podría ser de expansión), de acuerdo con la naturaleza específica de la enfermedad de que se trate y el desarrollo desigual de los avances terapéuticos y preventivos.

No obstante, desde el punto de vista epistemológico, la hipótesis de la compresión de la morbilidad cumple con los requisitos señalados por Sneed para aceptar una teoría como científica. ${ }^{7}$ En efecto, esta hipótesis contiene un conjunto de enunciados conceptuales y observacionales (que pueden clasificarse como propios y no propios); un conjunto de condiciones que permiten su ligadura; un conjunto de modelos potenciales de la teoría, y un conjunto de condiciones en donde ésta puede aplicarse.

Entre los enunciados conceptuales y observacionales que no son propios del modelo se encuentran los conceptos de edad de la muerte, esperanza de vida al nacimiento (y a otras edades), morbilidad, mortalidad y momento de inicio de la enfermedad. Estos enunciados, por su naturaleza de no-pertenencia al modelo teórico propuesto por la hipótesis, no requieren de un significado específico dentro de él, ya que son explicados por teorías exteriores al modelo. En cambio, los enunciados conceptuales propios del modelo poseen significado teórico sólo al interior de la hipótesis de la compresión; su importancia radica en las relaciones que establecen entre sí y con los enunciados que no son propios de la teoría pero que le proporcionan sustento empírico. Entre ellos destacan los de límite máximo de vida potencial, promedio máximo de vida potencial y compresión de la morbilidad. Las alternativas de expresión de la hipótesis son dos: la compresión y la expansión de la morbilidad poblacional.

\footnotetext{
* Gómez de León J, Frenk J. Population and health challenges in Mexico on the Eve of the 21st Century. Paper prepared for the meeting Choices for Health: Quantitative Principles for the Allocation of Resources in the Health Sector, ${ }_{1}^{1}$ organized by the World Bank; enero1992: Bali, Indonesia. Documento no publicado.
} 
Las condiciones en las cuales el modelo se aplica se establecen desde las premisas de la hipótesis y son, en resumen, las condiciones epidemiológicas de los países en los cuales la EVN ya rebasó los 70 años de edad.

Una ventaja importante del modelo es la posibilidad que ofrece para someter a contrastación sus consecuencias y, por ende, establecer su capacidad para describir, explicar o predecir el comportamiento de la morbilidad en grandes grupos poblacionales. Desde nuestro punto de vista, al poseer enunciados conceptuales y observacionales precisos y contrastables, la hipótesis de la compresión se coloca en condiciones epistemológicas superiores a las de otras teorías sobre la dinámica de la morbilidad, ya que estas últimas, al poseer insuficientes contenidos teóricos, han tenido que echar mano de múltiples hipótesis ad hoc para dar cuenta de sus fallas explicativas y predictivas. De hecho, al ser compatibles con la hipótesis de la compresión de la morbilidad, otras teorías pueden adquirir una mayor fortaleza teórica; éste es el caso de la teoría de la transición epidemiologica. ${ }^{37}$

Como queda claro, existe un conjunto de lagunas en donde el modelo de compresión de la morbilidad carece de potencia explicativa.$^{38}$ No obstante, es innegable que, de ser correcto, el modelo tendría una extraordinaria capacidad para predecir lo que sucederá con los eventos epidemiológicos y sanitarios en el futuro. Por esta razón, su desarrollo sistemático es de una importancia extraordinaria para la salud pública.

\section{Referencias}

1.Thuillier P. De Arquímedes a Einstein. Las caras ocultas de la invención científica. México, D.F.: Alianza Editorial, 1991.

2. Popper KR. Conjeturas y refutaciones. Madrid: Editorial Tecnos, 1967. 3. Popper KR. El conocimiento objetivo. Madrid: Editorial Tecnos, 1974. 4. Hanson N R. Patterns of discovery. Cambridge: Cambridge University Press, 1958

5. Rolleri JL. Introducción. En: Rolleri JL, ed. Estructura y desarrollo de las teorías científicas. México, D.F.: Universidad Nacional Autónoma de México, 1986:7-17.

6. Suppes P. ¿Q ué es una teoría científica?. En: Rolleri JL, ed. Estructura y desarrollo de las teorías científicas. México, D.F.: Universidad N acional Autónoma de México, 1986:167-178.

7. Sneed JD. Problemas filosóficos en la ciencia empírica de la ciencia: un enfoque formal. En: Rolleri JL, ed. Estructura y desarrollo de las teorías científicas. México, D.F.: Universidad Nacional Autónoma de México, 1986:179-214.

8. Kuhn T. La estructura de las revoluciones científicas. México, D.F.: Fondo de Cultura Económica, 1986.

9. W iener N. Cybernetics. N ueva York: W iley and Sons, 1948.

10. Graunt J. Natural and political observations made upon the bills of mortality. Baltimore: Johns Hopkins Press, 1939.

11. MacMahon B, Pugh TF. Epidemiology. Principles and methods. Boston: Little, Brown and Company, 1970.
12. Rose G, Baker DP.W hat is epidemiology? BMJ 1978;2:803-804

13. Almeida FN . Epidemiología sin números: una introducción a la ciencia de la epidemiología. W ashington, D.C.: O rganización Panamericana de la Salud, 1992.

14. Stallones RA.To advance epidemiology. Annu Rev Public Health 1980; 1:69-72.

15. Krieger $\mathrm{N}$. Epidemiology and the web of causation: has anyone seen the spider? Soc Sci Med 1994;39:887-903.

16. Buck C. Popper's philoso phy for epidemiologists. Int J Epidemiol 1975; 4:159-168.

17. Davies AM. C omments on "Popper's philosophy for epidemiologists", by C. Buck. Int J Epidemiol 1975;4:169-170.

18. Smith A. "Comments on Popper's philosophy for epidemiologists", by C. Buck. Int J Epidemiol 1975;4:171-172.

19. Jacobsen M.A gainst Popperized epidemiology. Int J Epidemiol 1976;5: 9-11.

20. Buck C. Popper's philosophy for epidemiologists (letter). Int J Epidemiol 1976;5:97-98.

21. Susser M. Choosing a future for epidemiology. II. From black box to Chinese boxes and eco-epidemiology. Am J Public Health 1996;86:674677.

22. Fries JF. Aging, natural death, and the compression of morbidity. $\mathrm{N}$ Engl J Med 1980;303:130-135.

23. C arnes BA, 0 Ishansky SJ. Evolutionary perspectives on human senescence. Pop Dev Rev 1993;19:793-806.

24. Palmore E. The promise and problems of longevity studies. En: Palmore y Jeffers, ed. Prediction of life span. Massachussets: Health Lexington Books, 1971:3-11.

25. Strehler B. Genetic and cellular aspects of life span prediction. En: Palmore y Jeffers, ed. Prediction of life span. Massachussets: Health Lexington Books, 1971:33-49.

26. McFarlan D. Libro Guinness de los records. Madrid: Producciones Jordán, 1991:17-18.

27. Gernez-Rieux Ch, Gervois M. Medicina preventiva, salud pública e higiene. México, D.F.: Editorial Limusa, 1989.

28. Fries JF.The compression of morbidity. Milbank Mem Fund Q Health Soc 1983;61:397-419.

29. Fries JF. Aging, illnes, and health policy: Implications of the compression of morbidity. Perspect Biol Med 1988;31:407-428.

30. Fries JF. Strategies for reduction of morbidity. Am J C lin N utr 1992;55 suppl 6:1257-1262.

31. Kalache A, Sen K. Ageing in developing countries. En: Pathy MS, ed. Principles and practice of geriatric medicine. 3a. edición. John W iley and Sons, 1998.

32. Fries JF, G reen L, Levine $S$. Health promotion and the compression of morbidity. Lancet 1989;1:481-483.

33. Leibson C, Ballard D,W hisnant J, Melton J. The compression of morbidity hypothesis: Promise and pitfalls in using record-linked data bases to assess secular trends in morbidity and mortality. Milbank Q 1992;70: 127-154.

34. Maddox G, Clark D, Steinhauser K. Dynamics of functional impairment in late adulthood. Soc Sci Med 1994;38:925-936.

35. Cornman JN. Q uestions for societies with "Third Age" populations. The extension of Life Working Group, The Gerontological Society of America. Acad Med 1997;72:856-862.

36.0 mran A.The epidemiologic transition. A theory of the epidemiology of population change. Milbank Mem Fund Q 1971;49:509-538.

37.0 mran A.The epidemiologic transition theory. A preliminary update. JTrop Ped 1983;29:305-316.

38. Hempel CG. Sobre la concepción estándar de las teorías científicas. En: Rolleri JL. Estructura y desarrollo de las teorías científicas. México, D.F.: Universidad N acional Autónoma México, 1986:141-166. 